\title{
Chronic Jet Lag Produces Cognitive Deficits
}

\author{
Kwangwook Cho, ${ }^{1,4}$ A. Ennaceur, ${ }^{1}$ Jon C. Cole, ${ }^{1,2}$ and Chang Kook Suh ${ }^{3}$ \\ ${ }^{1}$ Department of Psychology, University of Durham, Durham, DH1 3LE, United Kingdom, 2Department of Psychology, \\ University of Liverpool, Liverpool, L69 7ZA United Kingdom, '3Department of Biophysics and Physiology, College of \\ Medicine, Inha University, Incheon, 402-751 Republic of Korea, and ${ }^{4}$ Medical Research Council Centre for Synaptic \\ Plasticity, Department of Anatomy, Medical School, University of Bristol, Bristol, BS8 1TD, United Kingdom
}

Traveling across time zones causes disruption to the normal circadian rhythms and social schedules because of travelers' shift in time. As the endogenous circadian timing system adapts slowly to new time cues, the phase relationship between biological rhythms and external time cues are out of synchronization for a period of time. This disturbance of circadian rhythms has been shown to impair physical and psychological health (Winget et al., 1984). To test the effects of repeated jet lag on mental abilities, airline cabin crew were compared with ground crew. Salivary cortisol was used as a physiological marker for circadian disruption. The cabin crew group, who had a history of repeated jet lag, had significantly higher salivary cortisol levels in an average working day. In addition, this elevated level of cortisol was only seen in the same subjects when the cabin crew were on transmeridian flights but not domestic flights. Cabin crew also exhibited cognitive deficits, possibly in working memory, that became apparent after several years of chronic disruption of circadian rhythms.

Key words: jet lag; cortisol; stress; human subject; cognition; memory
Many studies have reported the physical and psychological effects of transmeridian flying (i.e., traveling across different time zones). Sleep disturbances have been reported in $\sim 78 \%$ of subjects during the first night after a transmeridian flight. However, only $30 \%$ of subjects report sleep disturbances after three nights. In the same study, $\sim 60 \%$ of the same subjects also reported gastrointestinal problems (Winget et al., 1984). It has also been shown that disruption of circadian rhythms induced by transmeridian flying is a stress factor that delays ovulation and causes menstrual disturbances in 30-35\% female aircrew (Iglesias et al., 1980). Wright et al. (1983) have shown that after a transmeridian flight, $50 \%$ of subjects reported fatigue and sleep disturbances, and $40 \%$ reported subjective weakness. There were decrements in both cognitive and physical performance, and some of the subjects were unable to even complete the tasks. In the same study, no performance decrements were found after a north-south flight. Thus, rapid time-zone transitions impaired performance and consequently the safety of aircrew (Winget et al., 1984).

Glucocorticoids (GCs) are produced by the physical and psychological stress-responsive hypothalamic-pituitary-adrenal axis. Increases in plasma or salivary cortisol levels are used as biochemical markers of stress, and normal cortisol levels have been shown to have a diurnal rhythm with a maximum level in the morning and a nadir during the night (Van Cauter et al., 1996; Lupien et al., 1997). Several recent reports indicate that GCs can regulate neuronal metabolism, gene expression, and hippocampal synaptic plasticity (McEwen et al., 1999; Newcomer et al., 1999).

\footnotetext{
Received Oct. 4, 1999; revised Jan. 18, 2000; accepted Jan. 18, 2000.

We thank Drs. E. C. Warburton, J. Crabtree, N. Kemp, and Prof. M. W. Brown for helpful comments on this manuscript. We also thank Drs. J. Wakely and C D. Ingram for discussion about the endocrinology system.

Correspondence should be addressed to Kwangwook Cho, Medical Research Council Centre for Synaptic Plasticity, Department of Anatomy, Medical School, University of Bristol, Bristol BS8 1TD, UK. E-mail; Kei.Cho@bris.ac.uk.

Copyright (C) 2000 Society for Neuroscience $\quad 0270-6474 / 00 / 200001-\bullet \$ 15.00 / 0$
}

Desir et al. (1981) found that jet lag did not produce quantitative changes in the secretion of cortisol, with no change in the mean level of cortisol over $24 \mathrm{hr}$ after one transmeridian flight. The temporal organization of the cortisol secretions was, however, disrupted by the flight, and full adaptation of the circadian rhythm in cortisol secretion took $11 \mathrm{~d}$. This study was performed in male travelers after one transmeridian flight, whereas typically female cabin crew make multiple transmeridian flights within this adaptation period (Suvanto et al., 1993).

In the present study, the effects of chronic jet lag on levels of salivary cortisol and memory function were examined in 62 normal women employed in international airline companies. Cortisol measurements were made over the working day by both cabin crew (weekly transmeridian flights) and ground crew (no regular transmeridian flights) to determine if regular exposure to jet lag increased cortisol secretion. Working memory performance and reaction times were measured under baseline conditions in both groups, who were also subdivided into career cohorts (1-4 years of service in their respective careers). This was to examine the hypothesis that exposure to regular disruption of circadian rhythms would cause cognitive decrements. To ensure that transmeridian flying was the cause of any alterations in cortisol levels,

This article is published in The Journal of Neuroscience, Rapid Communications Section, which publishes brief, peerreviewed papers online, not in print. Rapid Communications are posted online approximately one month earlier than they would appear if printed. They are listed in the Table of Contents of the next open issue of JNeurosci. Cite this article as: JNeurosci, 2000, 20:RC66 (1-5). The publication date is the date of posting online at www.jneurosci.org.

http://www.jneurosci.org/cgi/content/full/4030 
comparisons were made within subjects (cabin crew only) on both short-distance and long-distance international flights.

\section{MATERIALS AND METHODS}

Subjects. The subjects were 24 - to 29 -yr-old women $(n=62)$ who were all employees of international airline companies, right-handed, university graduates, and had no history of neurological or psychiatric illness. One group was chosen who had little or no circadian rhythm reset history (i.e., airport check-in counter staff), and the other group consisted of airline flight attendants (i.e., cabin crew) who had over $8 \mathrm{hr}$ of jet lag per week. According to their flight schedule, cabin crew crossed different time zones at least once per week, and they had 2-4 d break before their next flight duty. The subjects were divided into career cohorts based on the number of years that they had spent in their careers, from 1 to 4 years. All analysis was performed blind of the subject group. This study was conducted in accordance with the Declaration of Helsinki.

Procedure. Before starting the experiment, the subjects were fully informed of the aims of the study and how to respond to the tasks, and all subjects gave informed consent to participate in the experiment. Saliva samples were taken from the cabin crew on both long-distance international [i.e., traveling across more than $8 \mathrm{hr}$ different time zones: $>8$ Greenwich Mean Time (GMT)] and short-distance flights (i.e., traveling across $<3 \mathrm{hr}$ different time zones: $<3$ GMT) but from the ground crew on only one occasion. In all cases, the temporal continuity was maintained with GMT (all measurements were taken on outward flights, so the time was the same for both aircrew and ground crew).

Cortisol measurement. To analyze salivary cortisol levels, the technique of Lupien et al. $(1994,1997)$ was used. The subjects were asked to collect saliva samples at $1 \mathrm{hr}$ intervals during their working day, samples were collected once per week and averaged for the 2 month periods. After mouth rinsing, the filter paper (Whatman) was placed in their mouth until the saliva reached the front line of the paper (filter paper was prepared beforehand for the collection of saliva). The filter paper was then air-dried and stored at $-18^{\circ} \mathrm{C}$ until analysis. Cortisol was extracted from the filter paper in $2 \mathrm{ml}$ of ethanol for $1 \mathrm{hr}$ at room temperature $\left(20 \pm 1^{\circ} \mathrm{C}\right)$. A $300 \mu \mathrm{l}$ aliquot of the extract was assayed using $\left[{ }^{3} \mathrm{H}\right]$ cortisol as the radiotracer and a highly specific cortisol antibody (B-63 antibody; Endocrine Science, Tarzana, CA).

Memory tasks. The experimental tasks were conducted with an Apple Macintosh computer using Macintosh stimulus presentation software (SuperLab; Cedrus, Wheaton, MD) (Haxby et al., 1993). Delayed matchto-sample tasks were used to test memory performance. The task was given at 11:00 A.M. local time (i.e., $3 \mathrm{hr}$ after starting work). Each visual memory task started with a short $(2.5 \mathrm{sec})$ presentation of an identical pair of stimulus pictures, followed by the presentation $(5 \mathrm{sec})$ of a black dot in the center of the screen to hold the subject's attention, and then after a 5 or $25 \mathrm{sec}$ delay, the test picture was presented for $2.5 \mathrm{sec}$. Four different types of stimuli were used; symbol, meaningful word, and Arabic numerals. In the case of a word stimulus, the pair of words comprised a meaningful word pair (three to eight letters in length), and the pair of number stimulus pictures comprised one or two digits of Arabic numerals. Each subject was presented with a total of 80 trials (i.e., 20 items times four types of stimuli), and the different types of stimuli were presented in a pseudorandom order. After the test picture, the subject had to choose the correct response by pressing a key. If the test pair of pictures was exactly the same as the stimulus pair of pictures, the subject had to press one key, and if not, then they had to press another key. The next stimulus picture was presented after the subjects had pressed any response key after the preceding test picture. The computer recorded the number of correct responses and the reaction time of the subjects.

Statistical analysis. Statistical analysis was made by Student's nonpaired $t$ test and regression analyses. Data are presented as mean \pm SEM. $p<0.05$ was taken as the level of significance throughout all the analysis.

\section{RESULTS}

Flight attendants had significantly higher salivary cortisol levels than ground crew during their average working day on a transmeridian flight (cabin crew, $18.4 \pm 0.6 \mathrm{nmol} / \mathrm{l}$; ground crew, $14.4 \pm 0.3 \mathrm{nmol} / 1 ; t=6.2 ; p<0.0001$ vs ground crew; Fig. 1 ) but not on a short-distance flight (short-distance flight, $14.7 \pm 0.3$ $\mathrm{nmol} / \mathrm{l} ; t=0.8 ; p>0.5$ vs ground crew). Transmeridian flying

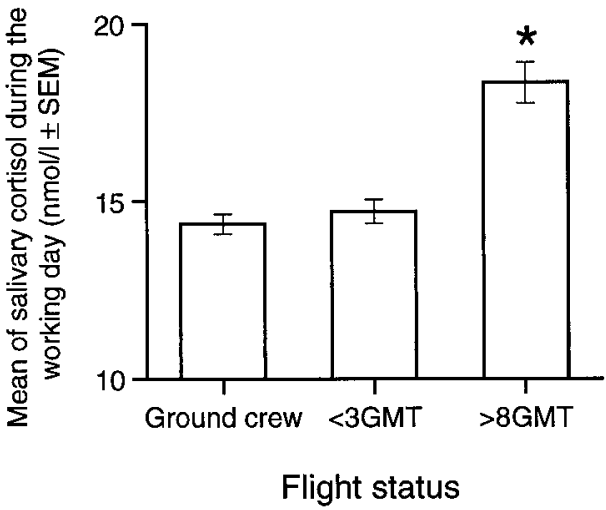

Figure 1. Salivary cortisol levels of cabin crew and ground crew. Longdistance international flight schedules $(>8$ GMT, $>8 \mathrm{hr}$ GMT different time zones flying) show higher cortisol level than ground crew and short-distance flight schedules $(<3$ GMT, $<3$ hr GMT different time zones flying). ${ }^{*} p<0.001$ versus ground crew and short-distance flight schedule (same long-distance international flight crew subjects). Error bars indicate SE $(n=10)$.

also significantly increased salivary cortisol levels over domestic flying in the cabin crew $(t=19.6 ; p<0.0001$ vs short-distance flight; Fig. 1). There was no significant difference in salivary cortisol levels because of the number of years of service. Finally cortisol levels were also monitored just after the memory performance task (ground crew, $13.7 \pm 0.5 \mathrm{nmol} / 1 ; n=10$; cabin crew, $15.8 \pm 0.7 \mathrm{nmol} / 1 ; n=10)$.

There was no significant difference in memory performance between cabin crew and ground crew with $<3$ years service (Fig. $2 A, B)$. However, cabin crew with 4 years of service had a significantly lower number of correct responses at both the $5 \mathrm{sec}$ (cabin crew, $85 \pm 2 \%$; ground crew, $91 \pm 2 \%$; $t=3.3 ; p<0.005$; Fig. $2 A$ ) and $25 \mathrm{sec}$ delay (cabin crew, $82 \pm 1 \%$; ground crew, $91 \pm 1 \%$; $t=$ 5.2; $p<0.0001$; Fig. 2B). The relationship between salivary cortisol level and percentage of correct responses was examined by regression analyses. A significant correlation was found in cabin crew $\left(r=-0.78, r^{2}=0.67, p<0.01 ; r=-0.75, r^{2}=0.57\right.$, $p<0.01 ; 5 \mathrm{sec}$ and $25 \mathrm{sec}$ delay, respectively; Fig. $2 C$ ), but there was no correlation in ground crew $\left(r=-0.43, r^{2}=0.19, p>0.1\right.$; $r=-0.34, r^{2}=0.12, p>0.1 ; 5 \mathrm{sec}$ and $25 \mathrm{sec}$ respectively, Fig. $2 D)$. The percentage of correct responses in subjects with high cortisol levels $(17-21 \mathrm{nmol} / \mathrm{l})$ was $<85 \%$ compared to a $95 \%$ performance level in the ground crew. In addition, the reaction time of cabin crew was significantly slower than that of ground crew with 4 years service (symbol, 5 sec delay: $t=2.8, p<0.05$; symbol, 25 sec delay: $t=4.4, p<0.005$; number, 25 sec delay: $t=$ $2.9, p<0.01$; Fig. $3 B$ ). There was no statistically significant difference in reaction time attributable to the number of years of service (1-3 years of service in their respective careers; Fig. 3A).

In contrast, cabin crew from other airlines (i.e., cabin crew who have more than a $14 \mathrm{~d}$ interval between outward transmeridian flights; Fig. $4 A$ ) had performance levels $>85 \%$ correct. Also in this group no memory deficits were found to be associated with transmeridian flying even though the flights increased salivary cortisol levels (salivary cortisol during the working day, 15-20 nmol/1; Fig. 4B,C). Finally, there was no significant correlation between cortisol levels and memory performance in this airline cabin crew $\left(r=-0.2, r^{2}=0.04, p>0.1 ; r=-0.12, r^{2}=0.01, p>\right.$ $0.1 ; 5 \mathrm{sec}$ and $25 \mathrm{sec}$ delay, respectively; Fig. $4 B$ ). 


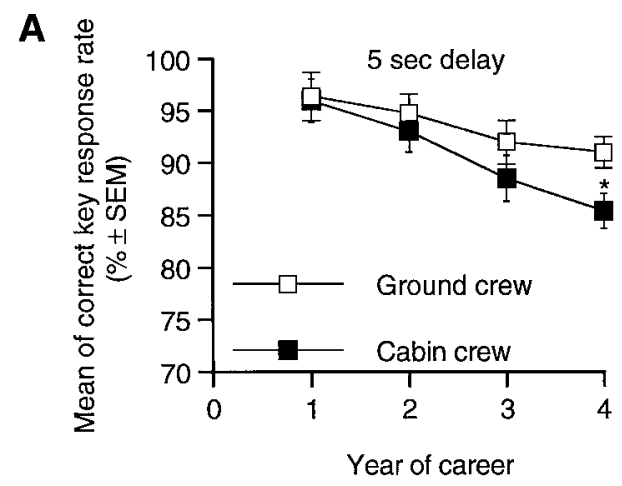

C

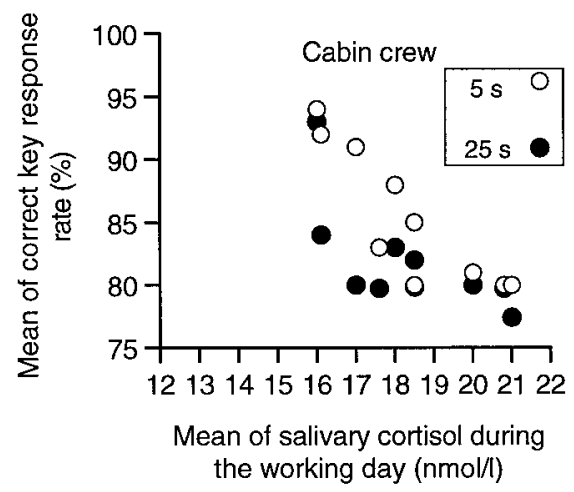

A

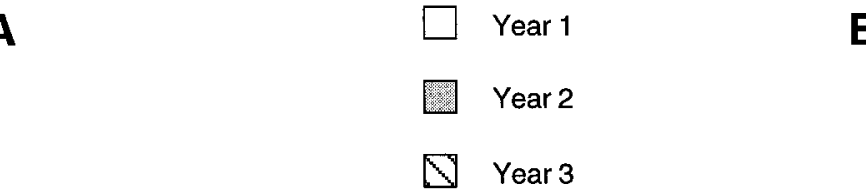

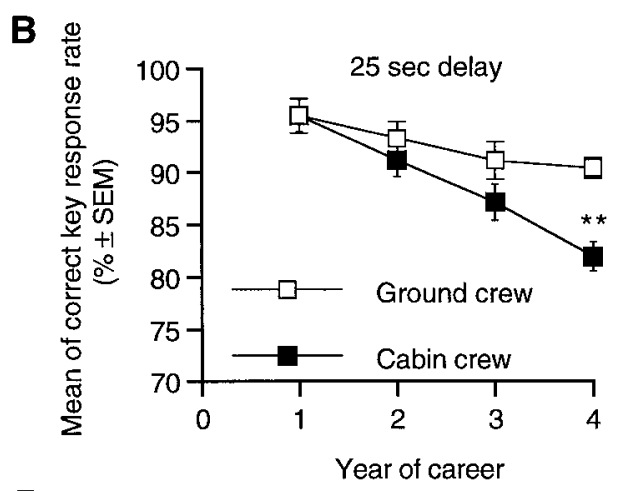

D

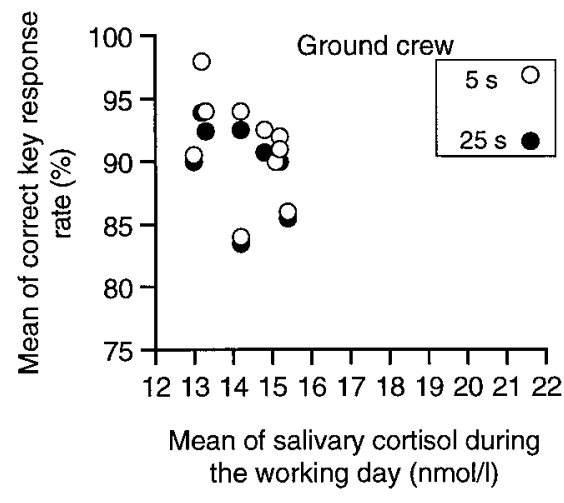

Figure 2. A, Percentage of correct key responses after $5 \mathrm{sec}$ delay test picture and after $25 \mathrm{sec}$ delay decreases across years in respective careers between ground crew and cabin crew $(B) .{ }^{*} p<$ 0.005 versus ground crew; $* * p<0.0001$ versus ground crew $(n=6$ in each year). $C$ and $D$ show the relationship between cortisol level and correct key response rate (cabin crew with 4 years service; $n=10)$.

\section{B}

\section{Year 4 ground crew}

Year 4 cabin crew
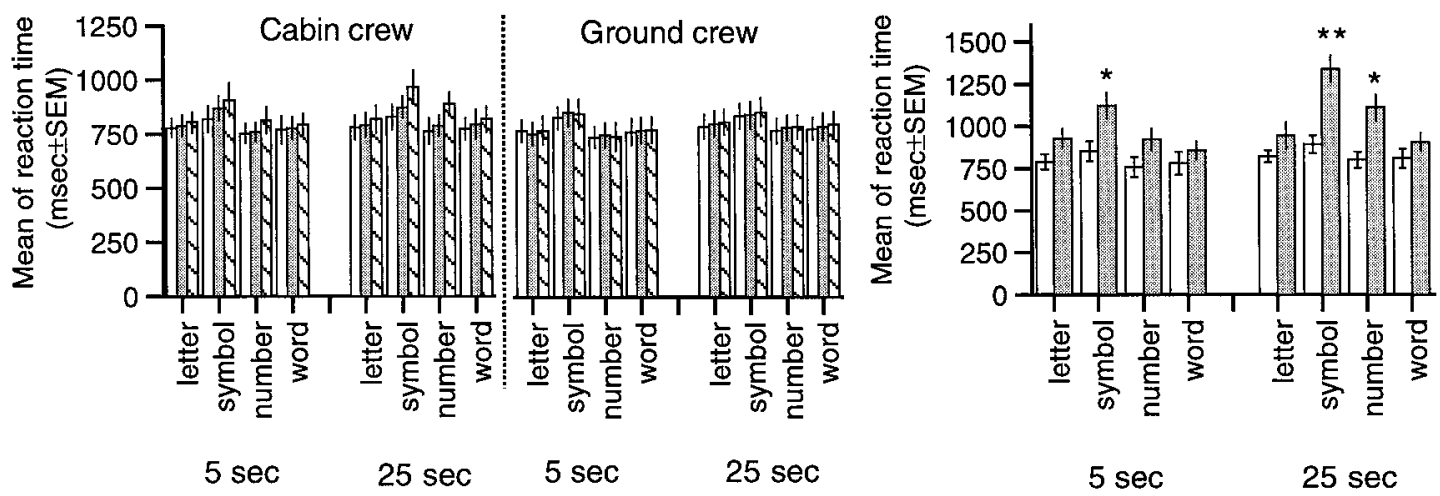

$5 \mathrm{sec}$

$25 \mathrm{sec}$

Figure 3. A, Reaction times were divided into career cohorts based on the number of years $(1-3$ years of service in their respective careers; $n=6$ in each year). $B$, Reaction time increases in cabin crew over ground crew with different stimulus pairs (4 years of service). ${ }^{*} p<0.01$ versus ground crew (symbol, $5 \mathrm{sec}$ delay, cabin crew: $1125 \pm 74 \mathrm{msec}$; symbol, $5 \mathrm{sec}$ delay, ground crew: $852 \pm 61 \mathrm{msec}$; number, $25 \mathrm{sec}$ delay, cabin crew: $1111 \pm 77 \mathrm{msec}$; number, $25 \mathrm{sec}$ delay, ground crew: $801 \pm 48 \mathrm{msec}$ ), ${ }^{* *} p<0.005$ versus ground crew (symbol, 25 sec delay, cabin crew: $1341 \pm 87 \mathrm{msec}$; ground crew: $891 \pm 55 \mathrm{msec})$. Error bars indicate SE $(n=10)$.

\section{DISCUSSION}

The present study showed that cabin crew had higher levels of salivary cortisol than ground crew across all four career cohorts tested. This increase was maintained across the working day and seems to suggest that flying increases the secretion of cortisol. In the present study, all the aircrew subjects had at least several transmeridian flights in the preceding weeks, which may account for the increased cortisol secretion in the aircrew subjects. This finding is in contrast to earlier reports of no increased cortisol secretion after transmeridian flying (Desir et al., 1981). The study of Desir et al. (1981), however, was conducted in subjects that had no circadian rhythm disruption in the previous year.

International transmeridian flying was associated with significantly raised salivary cortisol compared to short-distance flying in the same cabin crew subjects on the outward journey. This indicates that the increase in circulating cortisol was not caused by the effect of flying per se. It is possible, however, that anticipation of the deleterious effects of "jet lag" was stressful for these subjects, 
A

\begin{tabular}{|c|c|c|c|c|}
\hline+9 GMT & \multicolumn{3}{|c|}{$0 \mathrm{GMT}$} & $+9 \mathrm{GMT}$ \\
\hline Osaka & \multicolumn{3}{|c|}{ London (4 nights) } & Osaka \\
\hline \multicolumn{4}{|r|}{$+8 \mathrm{GMT}$} & +9 GMT \\
\hline \multicolumn{3}{|c|}{ Osaka (4 nights) } & $\begin{array}{l}\text { Dairen } \\
\text { Osaka }\end{array}$ & $\begin{array}{c}\text { Osaka } \\
\text { Domestic }\end{array}$ \\
\hline \multirow{2}{*}{\multicolumn{2}{|c|}{$\begin{array}{l}\text { Short-distance schedule } \\
\text { for } 10=14 \text { days }\end{array}$}} & \multicolumn{3}{|c|}{ O GMT } \\
\hline & & \multicolumn{3}{|c|}{ London (4 nights) } \\
\hline
\end{tabular}

Figure 4. Data from other airlines that have long intervals between transmeridian flights. $A$ shows typical cabin crew flight duty schedule. $B$ shows that the relationship between salivary cortisol level and correct key response rate (mean of salivary cortisol: $17 \mathrm{nmol} / 1, t=$ $1.9, p>0.05$ vs cabin crew; Fig. $2 C ; 5 \mathrm{sec}$ delay average correct key response rate: $92 \%, t=3.4, p<0.005$ vs cabin crew; Fig. $2 C ; 25 \mathrm{sec}$ delay average correct key response rate: $90 \%, t=4.6, p<0.001 \mathrm{vs}$ cabin crew; Fig. $2 C, n=10$ ). $C$, Reaction time with four different types of stimulus pairs (all stimulus pairs: $p>$ 0.05 vs ground crew; Fig. $3, n=10$ ).
B

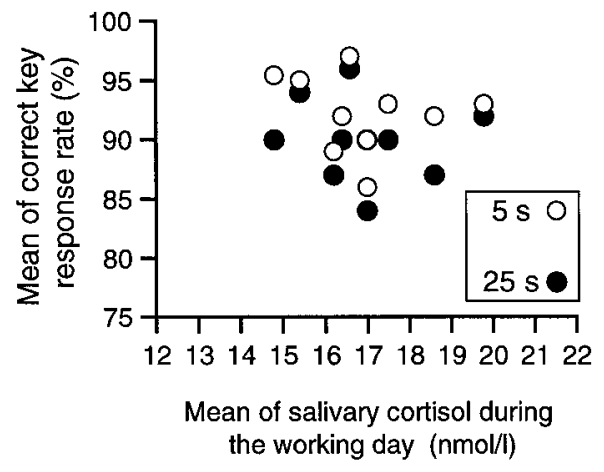

C

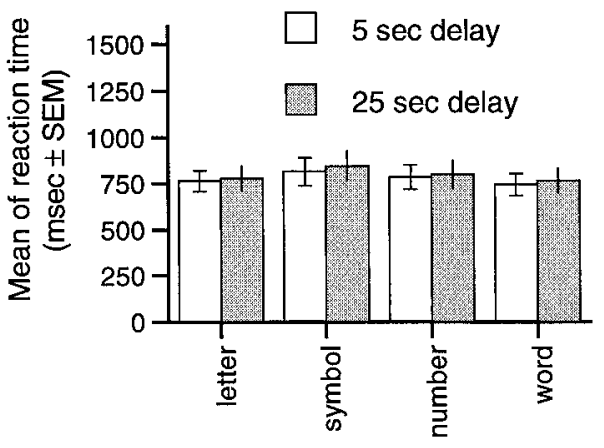

and this caused the increase in cortisol secretion before the circadian disruption. This effect was observed across all four career cohorts, which suggests that it is not something to which the subjects were able to adapt. One might have expected that after 4 years of flight experience, the subjects would have adopted a successful strategy for reducing the negative impact of flying.

Recent evidence has shown that chronic exposure to high levels of circulating corticosteroids impairs cognitive function in both animals and humans (Lupien et al., 1994, 1997, 1998; McEwen and Sapolsky, 1995). After 4 years of transmeridian flying experience, the aircrew showed statistically significant correct key rate and reaction time deficits. The delayed onset of this decrement implies that exposure to the elevated level of cortisol for 4 years had impaired these functions a result, which is in line with previous findings (Lupien et al., 1994, 1997; McEwen and Sapolsky, 1995). Years of career also appeared to negatively affect the ground crew's cognitive performance as well. This decline may be explained by age-related influences, however, further study is required to confirm this (Grady et al., 1998; Hazlett et al., 1998).

As the results showed, the cognitive deficits were found to be dependent on both delay interval and the type of stimuli used. In case of symbol test picture, the correct key response latency was $>1 \mathrm{sec}$ in cabin crew at both the 5 and $25 \mathrm{sec}$ delay. The cabin crew also showed significantly longer reaction times when the number test picture was presented, but only in the $25 \mathrm{sec}$ delay condition. Thus, cognitive deficits were evident in both the symbol and the number test conditions, but not when the letter or word test pictures were presented. These results suggest that jet lag may depress nonverbal short-term memory processing. It may also be argued that the performance decrements observed in the present study may be the result of an attentional deficit. Both the reaction time and correct number of responses were differentially affected by delay time and the types of test picture. However, the performance deficit is found in both the $5 \mathrm{sec}$ delay symbol test condition and the $25 \mathrm{sec}$ delay condition. Therefore, this may suggest that performance deficits are a result of impairments in nonverbal cognitive processing. Both cabin crew and ground crew showed longer reaction times in the symbol test condition but not the other test conditions. Thus, these stimuli may be more difficult to recognize than other test conditions. Further studies are needed to determine the particular types of cognitive deficits produced by long-term chronic jet lag.

In cabin crew from other airlines tested, no memory deficits were shown to be associated with transmeridian flying, even though the flights increased salivary cortisol levels (Fig. 4B,C). One possible explanation for these findings is that these other airlines have longer intervals between transmeridian flights (i.e., cabin crew have an interval between outward long-distance flights of $>14 \mathrm{~d}$ ). This interval is a sufficient to allow the circadian rhythms to recover (Fig. $4 A$ ), and this may be a potential method of eliminating the cognitive deficits induced by jet lag. Jet lag causes sleep deprivation, and sleep deprivation itself may be a factor that will modulate attention and cognitive performance. However, previous studies have shown that short-term sleep deprivation $(<36 \mathrm{hr})$ does not selectively impair higher cortical functioning (Binks et al., 1999).

Newcomer et al. (1999) have demonstrated decreased memory performance induced by stress level cortisol treatment in healthy humans. The present study, therefore, demonstrates that jet lag produced by short time intervals between outward transmeridian flights elevates salivary cortisol levels and that long-term exposure to high cortisol levels may result in cognitive deficits, and in particular memory impairment. However, we cannot yet confirm whether these deficits are temporary or permanent. Lupien et al. (1998) have shown that basal cortisol elevation can cause hippocampal damage and impair hippocampal-dependent learning and memory. Thus, functional brain scanning may help to address these unsolved questions.

\section{REFERENCES}

Binks PG, Waters WF, Hurry M (1999) Short-term total sleep deprivations does not selectively impair higher cortical functioning. Sleep 22:328-334.

Desir D, Van Cauter E, Fang VS, Martino E, Jadot C, Spire J-P, Noël P, Refetoff S, Copinschi G, Goldstein J (1981) Effect of jet lag on hormonal patterns. I. Procedures, variations in total plasma-proteins, and 
disruption of adrenocorticotropin-cortisol periodicity. J Clin Endocrinol Metab 52:628-641.

Grady CL, McIntosh AR, Bookstein F, Horwitz B, Rapoport SI (1998) Age-related changes in regional cerebral blood flow during working memory for faces. NeuroImage 8:409-425.

Haxby JV, Parasuraman R, Lalonde F, Abboud H (1993) Superlab: general purpose software for human experimental psychology and psychological testing. Behav Res Methods Instrum Comput 25:400-405.

Hazlett EA, Buchsbaum MS, Mohs RC, SpiegelCohen J, Wei TC, Azueta R, Haznedar MM, Singer MB, Shihabuddin L, LuuHsia C, Harvey PD (1998) Age-related shift in brain region activity during successful memory performance. Neurobiol Aging 19:437-445.

Iglesias R, Terres A, Chavarria A (1980) Disorder of the menstrual cycle in airline stewardesses. Aviat Space Environ Med 51:518-520.

Lupien S, Lecours AR, Lussier I, Schwartz G, Nair NPV, Meaney MJ (1994) Basal cortisol levels and cognitive deficits in human aging. J Neurosci 14:2893-2903.

Lupien SJ, Gaudreau S, Tchiteya BM, Maheu F, Sharma S, Nair NPV, Hauger RL, McEwen BS, Meaney MJ (1997) Stress-induced declarative memory impairment healthy elderly subjects: relationship to cortisol reactivity. J Clin Endocrinol Metab 82:2070-2075.

Lupien SJ, de Leon M, de Santi S, Convit A, Tarshish C, Nair NPV, Thakur M, McEwen BS, Hauger R, Meany MJ (1998) Cortisol levels during human aging predict hippocampal atrophy and memory deficits. Nat Neurosci 1:69-73.

McEwen BS, Sapolsky RM (1995) Stress and cognitive function. Curr Opin Neurobiol 5:205-216.

McEwen BS, de Leon MJ, Lupien SJ, Meaney MJ (1999) Corticosteroids, the aging brain and cognition. Trends Endocrinol Metab 10:92-96.

Newcomer JW, Selke G, Melson AK, Hershey T, Craft S, Richards K, Anderson AL (1999) Decreased memory performance in healthy humans induced by stress-level cortisol treatment. Arch Gen Psychiatry $56: 527-533$.

Suvanto S, Harma M, Ilmarinen J, Partinen M (1993) Effect of 10h time zone changes on female flight attendants' circadian rhythms of body temperature, alertness and visual search. Ergonomics 36:613-625.

Van Cauter E, Leproult R, Kupfer DJ (1996) Effects of gender and age on the levels and circadian rhythmicity of plasma cortisol. J Clin Endocrinol Metab 81:2468-2473.

Winget CM, DeRosha CW, Markley CL, Holley DC (1984) A review of human physiological and performance changes associated with desynchronosis of biological rhythms. Aviat Space Environ Med 55:1085-1096.

Wright JE, Vogel JA, Sampson JB, Knapik JJ, Patton JF, Daniels WL (1983) Effects of travel across time zone (jet-lag) on exercise capacity and performance. Aviat Space Environ Med 54:132-137. 